\title{
The analysis of estrogen receptor- $\alpha$ positive breast cancer stem-like cells unveils a high expression of the serpin proteinase inhibitor PI-9: Possible regulatory mechanisms
}

\author{
MARIANNA LAURICELLA ${ }^{1}$, DANIELA CARLISI ${ }^{1}$, MICHELA GIULIANO ${ }^{2}$, GIUSEPPE CALVARUSO ${ }^{2}$, \\ CESARE CERNIGLIARO $^{2}$, RENZA VENTO $^{2}$ and ANTONELLA D'ANNEO ${ }^{2}$
}

Departments of ${ }^{1}$ Experimental Biomedicine and Clinical Neurosciences and ${ }^{2}$ Biological, Chemical and

Pharmaceutical Sciences and Technologies, Laboratory of Biochemistry, University of Palermo, Palermo, Italy

Received January 11, 2016; Accepted February 18, 2016

DOI: 10.3892/ijo.2016.3495

\begin{abstract}
Breast cancer stem cells seem to play important roles in breast tumor recurrence and endocrine therapy resistance, although the underlying mechanisms have not been well established. Moreover, in some tumor systems the immunosurveillance failure against cancer cells has been related to the presence of the granzyme B inhibitor PI-9. This study explored the status of PI-9 in tumorspheres isolated from estrogen receptor- $\alpha$ positive $\left(E R \alpha^{+}\right)$breast cancer MCF7 cells. Studies were performed in tertiary tumorspheres which possess high levels of stemness markers (Nanog, Oct $3 / 4$ and Sox 2 ) and selfrenewal ability. The exposure to estrogens (17- $\beta$ estradiol and genistein) increased the number and sizes of tumorspheres, promoting cell proliferation as demonstrated by the increase in the proliferating cell nuclear antigen (PCNA). The study of the three isoforms $(66,46$ and $36 \mathrm{kDa})$ of ER $\alpha$ disclosed that tertiary tumorspheres exhibit a marked increase in ER $\alpha 36$, while the level of ERa66, which is highly expressed in MCF7 cells, declines. Although it is known that PI-9 is a transcriptional target of ER $\alpha 66$, surprisingly in tertiary tumorspheres, despite the reduced level of ER $\alpha 66$, the protein and mRNA content of PI-9 is higher than in MCF7 cells. Treatment with estrogens further increased PI-9 level while decreased that of ER $\alpha 66$ isoform thus excluding the involvement of this receptor isoform in the event. Moreover, our studies also provided evidence that tertiary tumorspheres express elevated levels
\end{abstract}

Correspondence to: Dr Antonella D'Anneo, Department of Biological, Chemical and Pharmaceutical Sciences and Technologies, Laboratory of Biochemistry, University of Palermo, via del Vespro 129, 90127 Palermo, Italy

E-mail: antonella.danneo@unipa.it

Abbreviations: $\mathrm{BC}$, breast cancer; ER, estrogen receptor; CTL, cytotoxic T lymphocytes; NK, natural killer; PCNA, proliferating cell nuclear antigen; PI-9, proteinase inhibitor 9

Key words: serpin proteinase inhibitor 9, breast cancer stem-like cells, breast cancer, estrogen receptors of CXCR4 and phospho-p38, suggesting that the high PI-9 content might be ascribed to the activation of the proliferative CXCR4/phospho-p38 axis. Taken together, these events could supply a selective advantage to breast cancer stem cells by interfering with immunosurveillance systems and open up the avenue to new possible targets for breast cancer treatment.

\section{Introduction}

Breast cancer (BC) is the most common endocrine tumor and the second leading cause of cancer-related death in women (1). Approximately $75 \%$ of $\mathrm{BC}$ cases is characterized by the presence of hormone receptors (ERs) whose expression is considered an important prognostic and therapeutic indicator (2-4). ERs are nuclear receptors that regulate the transcription of estrogen-responsive genes in target cells after ligand binding and dimerization $(5,6)$. Moreover, it became evident that other pathways can be activated by ERs that involve cytoplasmic signaling proteins, growth factor receptors or other membrane signaling circuits (7). There are two ER types, $\mathrm{ER} \alpha$ and $\mathrm{ER} \beta$ which are responsible for both the canonical genomic and the non-genomic rapid pathways (8). In 2005 Wang et al identified and cloned a novel isoform of full-length ER $\alpha 66$ with a molecular weight of $36 \mathrm{kDa}$ (ER $\alpha 36)$ which is characterized by the lack of transcriptional activation domains and retains a partial ligand-binding domain (9). Although the prevalent subcellular localization of ER $\alpha 36$ (cytosol and cell membrane) and the lack of intrinsic transcriptional activity associate ER $\alpha 36$ to a non-genomic rapid estrogen signaling (10), the interplay between the diverse variants of ERs has not been fully established so far. It has been demonstrated that ER $\alpha 36$ can act as a dominant-negative regulator of signaling mediated by ER $\alpha 66$ but, on the other hand, the ER $\alpha 36$ expression seems to be subjected to an ERa66-dependent negative regulation (11). The presence of ERs in breast cancer cells allows the employment of ER antagonists, such as 4-hydroxytamoxifen, which are able to block their proliferative rate (12). However, although great strides have been made in BC therapy in recent years, $30-50 \%$ of cases of ER-positive BC still displays de novo or intrinsic resistance to ER antagonists and frequently tumor recurrence is observed $(13,14)$. 
There is substantial evidence that the main cause of cancer relapse and chemoresistance is the presence of cancer stem cells (CSCs) in tumor tissues (15). CSCs represent a small population within the tumor mass that not only drives tumor initiation and growth, but also mediates tumor metastasis and therapy resistance. They are very hard to eradicate due to their robust survival mechanisms. Although many proliferative pathways were identified in BC stem-like cells obtained from cancer tissues or cell lines, the role of estrogen signaling has not been fully established and the expression of ER $\alpha 66$ still remains controversial. Recently, Deng et al found that stem cells derived from ER-positive BC cells express high levels of ER $\alpha 36$ that play important roles in mediating anti-estrogen resistance (16).

It has been largely demonstrated that the malignancy of tumor cells is also sustained by the ability to escape immunosurveillance controlled by immune system cells such as cytotoxic T lymphocytes (CTL) and natural killer (NK) cells that recognize and kill cancer cells (17). Immune system cells eradicate tumor cells through two different death pathways: the perforin/granzyme B and Fas/Fas ligand pathway (18). In perforin/granzyme B-mediated apoptosis, the recognition of a target cell triggers the release of granules containing perforin and the granzyme protease. Perforin promotes granzyme delivery to the cytosol of target cell and, on entry, this protease triggers rapid and efficient apoptosis by the direct cleavage of caspase and non-caspase substrates (19). The activity of granzyme B, the main effector molecule of CTL and NK cells, is regulated by PI-9 (proteinase inhibitor 9), a member of the serine proteinase inhibitor family collectively called serpins (20). PI-9 is a pseudo-substrate for granzyme B leading to the irreversible inhibition of protease upon interaction (20).

We demonstrated that tumorspheres derived from ER $\alpha$-positive BC MCF7 cells express a high level of PI-9, supporting a role for the granzyme $\mathrm{B}$ inhibitor in immunosurveillance escape of $\mathrm{BC}$ stem cells.

\section{Materials and methods}

Cell culture and reagents. MCF7 cells were purchased from Istituto Scientifico Tumori (Genoa, Italy) and were maintained in Dulbecco's modified Eagle's medium (DMEM) supplemented with $5 \%$ heat-inactivated fetal bovine serum (FBS), $2 \mathrm{mM}$ glutamine, $50 \mathrm{U} / \mathrm{ml}$ penicillin and $50 \mu \mathrm{g} / \mathrm{ml}$ streptomycin at $37^{\circ} \mathrm{C}$ in a humidified atmosphere containing $5 \% \mathrm{CO}_{2}$. For estrogen challenge, 4-5 days before the experiments medium was replaced with phenol red-free DMEM containing 5\% charcoal-stripped FBS (Life Technologies, Monza, Italy). All reagents, except where differently reported, were purchased from Sigma-Aldrich (Milan, Italy).

Tumorsphere production. For tumorsphere preparation, $10^{4}$ MCF7 cells were seeded in ultra low-attachment 6-well plates in a conditioned medium bereft of serum and containing phenol red-free DMEM/F12, supplemented with 1X B27, $20 \mathrm{ng} / \mathrm{ml}$ EGF, $20 \mathrm{ng} / \mathrm{ml}$ bFGF (Life Technologies). Growth factors were added every two days (without removing the old media) and in these conditions cells grew as non-adherent spherical clusters named tumorspheres. Formed spheres were visualized under a bright-field Leica DMR inverted microscope (Wetzlar, Germany) and their sizes and number were measured and counted using IM50 Leica software (Wetzlar, Germany).

Tumorsphere passage. For sphere passage experiments, the tumorspheres were collected by centrifugation and dissociated by means of enzymatic ( $0.25 \%$ trypsin-EDTA) and mechanical treatment. After centrifugation to remove the enzyme, pellets were resuspended in serum starved conditioned medium to allow tumorsphere re-growth. The dissociated single sphereforming cells were diluted to a density of 1,000 cells $/ \mathrm{ml}$ and culture passaged every 7 days according to Dontu et al (21). Sphere formation efficiency (SFE) was calculated dividing the number of tumorspheres by the number of seeded cells.

Cell viability. To determine cell viability after estrogen treatments, MTT (3-(4,5-dimethylthiazol-2-yl-2,5-diphenyl tetrazolium bromide) assay was performed as previously reported (22). Briefly, $20 \mu \mathrm{l}$ of MTT $(11 \mathrm{mg} / \mathrm{ml})$ was added to the cells and incubation was protracted for $2 \mathrm{~h}$ at $37^{\circ} \mathrm{C}$. Then, medium was replaced with $100 \mu \mathrm{l}$ lysis buffer (20\% SDS, $10 \%$ dimethylformamide and $20 \%$ acetic acid) to lysate the cells. After solubilisation, the absorbance was read at 570 and $690 \mathrm{~nm}$. Each condition was tested six times and results were analyzed for statistical significance.

RNA extraction and $q P C R$ analysis. Total RNA was prepared using Direct-zol ${ }^{\mathrm{TM}}$ RNA MiniPrep kit (Zymo Research, Irvine, CA, USA). Then, after removal of residual genomic DNA with DNase I (Zymo Research), oligo(dT)-primed reverse transcription was performed on $1 \mu \mathrm{g}$ of total cellular RNA using the iScript ${ }^{\mathrm{TM}}$ cDNA Synthesis kit (Bio-Rad Laboratories Srl, Milan, Italy), following the manufacturer's instructions. For real-time PCR analyses, each cDNA sample was amplified by $\mathrm{iQ}^{\mathrm{TM}}$ SYBR Green Supermix (Bio-Rad), using the following QuantiTect primers (Qiagen, Milan, Italy): Oct3/4 (POU5F1: QT00210840), Sox2 (QT00237601), Nanog (QT01025850), Kfl4 (QT00061033), CXCR4 (QT00223188). As for PI-9 amplification, the following primers were used: Fw 5'-CGCTGGACTAGGTGGCAG-3' and Rv 5'-CAGAA CACGTTGTGCGAAGG-3' (Life Technologies).

All PCR reactions were performed in triplicate in the iQ5 thermal cycler instrument (Bio-Rad, Laboratories Srl, Milan, Italy), using a PCR cycling as previously reported (23). The relative quantification of analyzed genes was calculated using the $2^{-\Delta \Delta C t}$ method and data were normalized by using GAPDH (QT01192646; Qiagen) as endogenous control. Data processing and statistical analysis were performed by $\mathrm{iQ}^{\mathrm{TM}}$ Optical System software (Bio-Rad).

Western blot analyses. The procedure of western blot analysis was performed as previously described (24). Briefly, proteins (30 $\mu \mathrm{g})$ were separated by SDS-polyacrylamide gel electrophoresis and transferred to a nitrocellulose membrane (Bio-Rad) for detection with primary antibodies. All antibodies were from St. Cruz Biotechnology (St. Cruz, CA, USA) except for p38 and phospho-p38 (Cell Signaling Technology, Beverly, MA, USA). Then, filters were incubated with horseradish-peroxidase-conjugated secondary antibodies and the detections were developed by ECL Plus ${ }^{\mathrm{TM}}$ western blotting reagents (Amersham, GE Healthcare Life Science, Milan, Italy) according to the manu- 


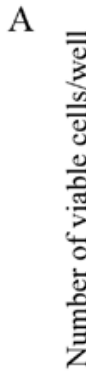

B
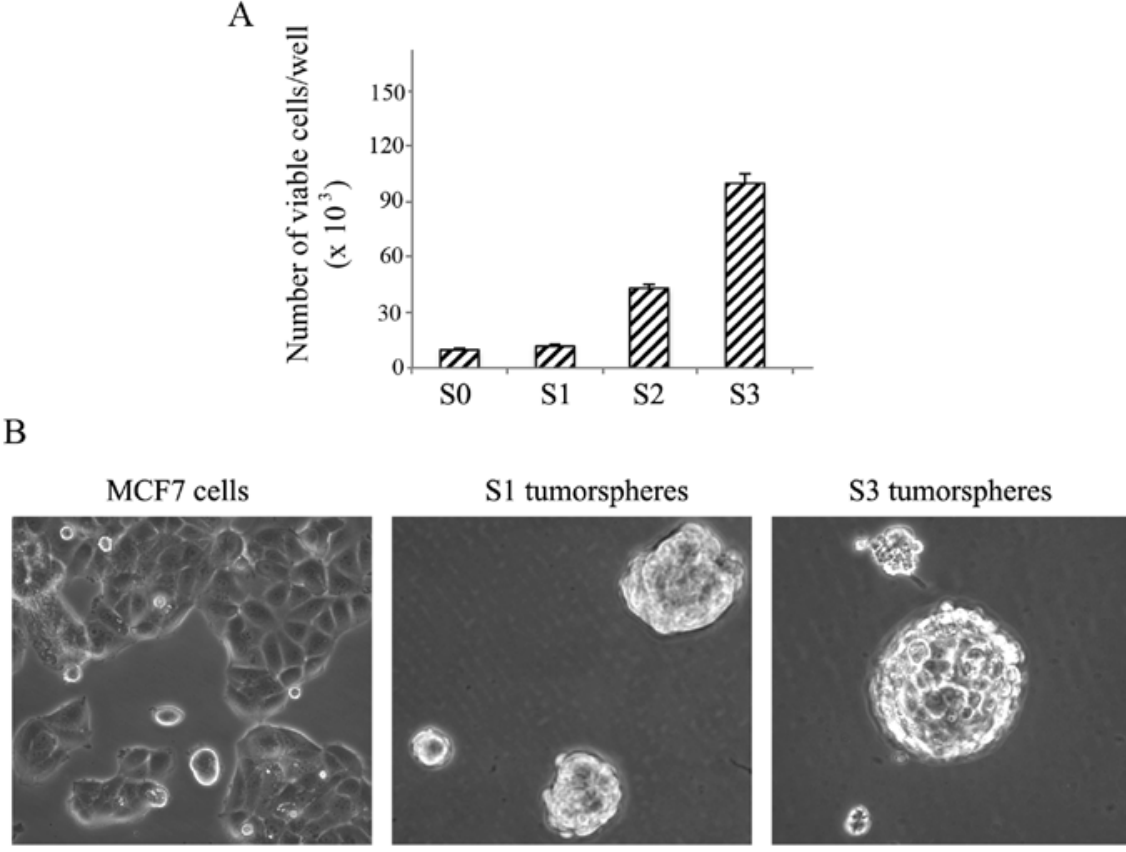

C
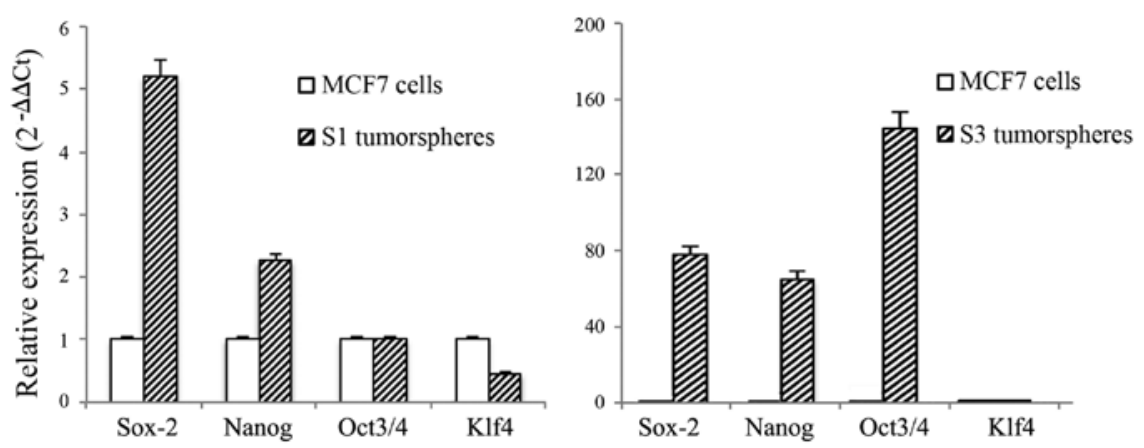

Figure 1. Morphological features and biochemical characterization of breast cancer derived tumorspheres. (A) Analysis of self-renewal ability. Sphere formation efficiency of serially passaged cells cultured as tumorspheres up to three generations was analyzed. After each serial passage, the number of viable dissociated cells was calculated at each generation and plotted against passage number. (B) Representative phase contrast images of adherent MCF7 cells, primary (S1) and tertiary (S3) tumorspheres obtained, respectively, after 7 and 21 days of culturing in a conditioned medium in ultra-low adherent plates. Micrographs were taken by a Leica microscope equipped with a computer-imaging system. The original magnification was at x100. (C) Real-time PCR analysis of stemness markers in parental MCF7 cells and breast cancer tumorspheres. Total RNA from MCF7 cells, S1 and S3 tumorspheres was reverse transcribed and amplified with appropriate primers in iQ5 cycler instrument as described in Materials and methods. Histograms report relative amounts of Sox 2, Nanog, Oct3/4 and Kfl4 to the amount of GAPDH calculated from data generated by iQ5 software package. The results are the means of three independent experiments.

facturer's instructions. Bands were visualized and acquired by ChemiDoc XRS System (Bio-Rad, Hercules, CA, USA).

Densitometric analysis of the bands was performed using the NIH Image J 1.40 analysis software distributed by National Institutes of Health (Bethesda, MD, USA). The correct protein loading was ascertained by both red Ponceau staining and immunoblotting for $\beta$-actin. All the blots shown are representative of at least three separate experiments.

Statistical analysis. Data, reported as means \pm SD from at least three independent experiments, were analyzed using the Student's t-test. Differences were considered significant at $\mathrm{P}<0.05$.

\section{Results}

Isolation and characterization of ERQ-positive breast cancer tumorspheres. In our study we first isolated and character- ized tumorspheres derived from adherent $\mathrm{ER} \alpha^{+} \mathrm{MCF} 7$ breast cancer cells to individuate new selective targets in BC stem cells. To this purpose MCF7 cells were seeded in ultra-low attachment plates and maintained in serum-starved medium to form non-adherent spherical clusters named tumorspheres. These spheres were already visible after 4-5 days and, when they reached a diameter $>50 \mu \mathrm{m}$ (approximately after 7 days), were serially passaged for different generations. Single cells obtained from sphere dissociation were propagated forming again new tumorspheres with a sphere formation efficiency (SFE) $2 \%$. The morphological and molecular features of tumorspheres were analyzed after 7 (primary spheres, S1), 14 (secondary spheres, S2) and 21 (tertiary spheres, S3) days, respectively. As shown in Fig. 1A, the number of viable cells, calculated and plotted against the equivalent passage number, progressively increased at each generation. In Fig. 1B the characteristic morphological aspects of S1 and S3 tumorspheres 
A

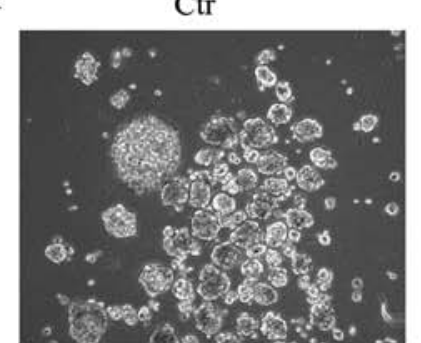

E2

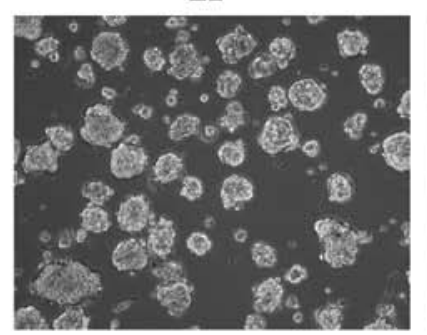

genistein

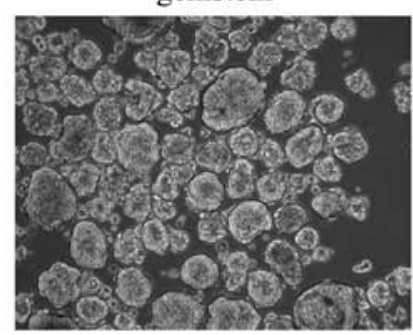

B

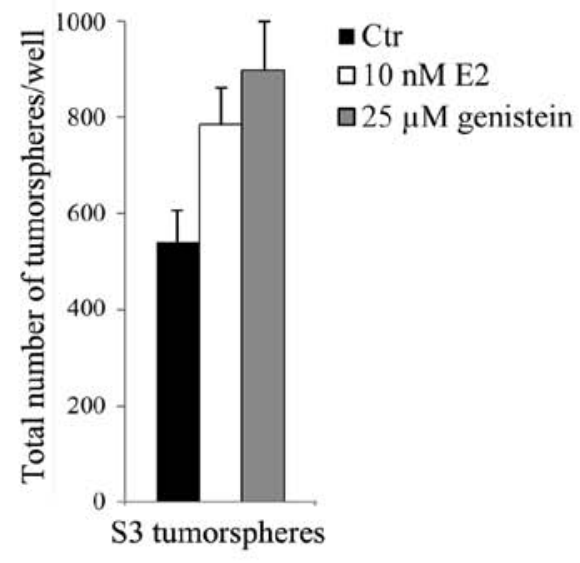

$\mathrm{C}$

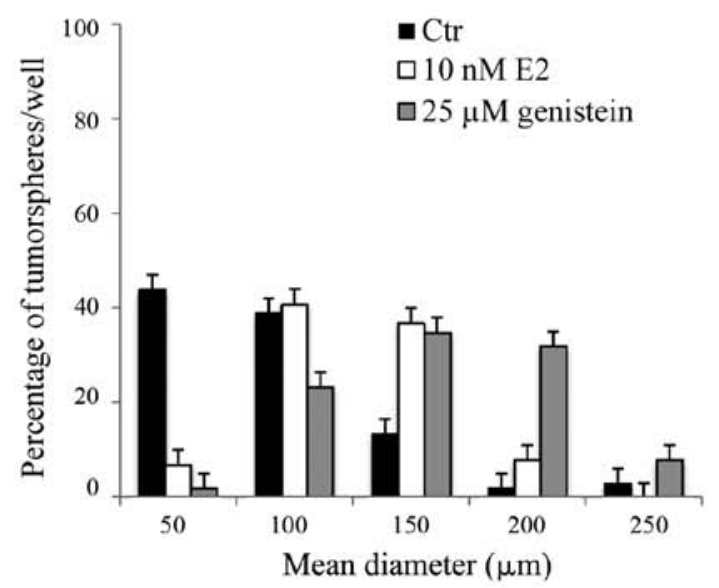

D

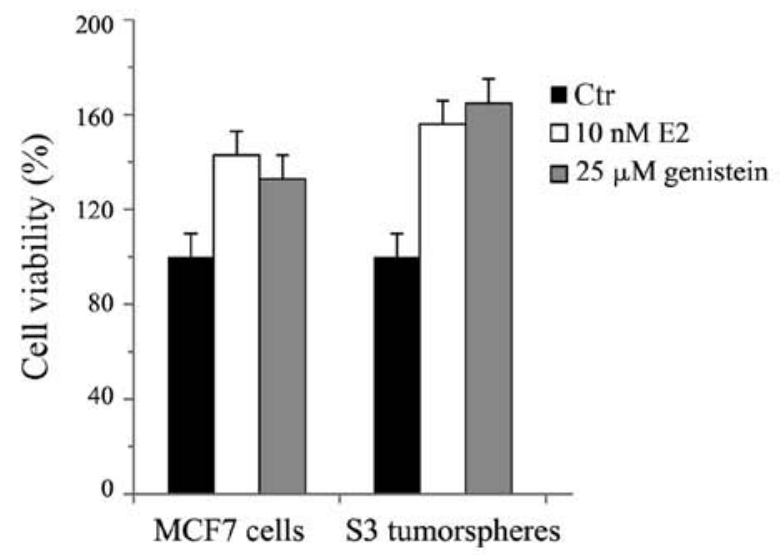

E

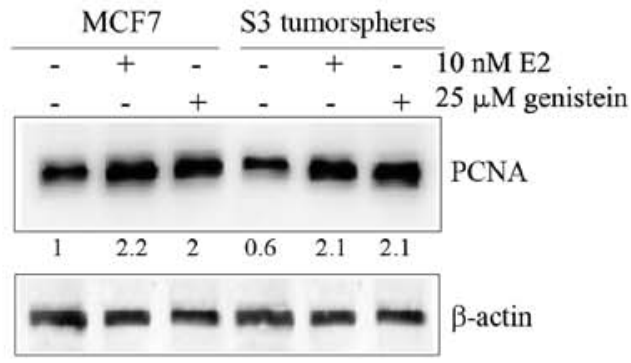

Figure 2. Analysis of proliferating effects of 17- $\beta$ estradiol and genistein on S3 tumorspheres. (A) Micrographs of S3 tumorspheres cultured in low-attachment plates and treated with $10 \mathrm{nM} 17-\beta$ estradiol (E2) or $25 \mu \mathrm{M}$ genistein for $48 \mathrm{~h}$. Micrographs at original x100 magnification are reported. (B and C) Effect of E2 and genisten on number (B) and size (C) of S3 tumorspheres. The total number of tumospheres and the corresponding diameters were calculated as reported in Materials and methods. (D) Effects of E2 and genistein on S3 tumorsphere cell viability in comparison to MCF7 cells. Cells were treated with 10 nM E2 or $25 \mu \mathrm{M}$ genistein for $48 \mathrm{~h}$; then MTT assay was performed as reported in Materials and methods. (E) Western blot analysis of PCNA in parental MCF7 cells and S3 tumorspheres. In (B-D) values are the means of three independent experiments \pm SD.

grown in anchorage-independent conditions are reported. As shown, both S1 and S3 tumorspheres grew in spheroidal clusters floating in the medium and were deprived of the flattened cell morphology, typical of adherent parental MCF7 cells.

Next, tumorspheres were collected by centrifugation and characterized by real-time RT-PCR to evaluate changes in the gene expression of stemness markers such as Sox 2, Nanog, Oct3/4, and Kfl4. The analysis reported in Fig. 1C shows an increase in the levels of Sox 2 and Nanog stemness markers in $\mathrm{S} 1$ tumorspheres with respect to parental MCF7 cells. The gene expression of these markers consistently increased in S3 tumorspheres where a remarkable upregulation of Oct3/4 was also observed (Fig. 1C). Our data also provided evidence that no significant change was present in the content of Kfl4. In light of these results, since $S 3$ tumorspheres exhibited the highest content of the examined stemness markers, we performed the next experiments in these tumorspheres comparing data to parental MCF7 cells.

Effects of 17- $\beta$ estradiol and genistein in adherent MCF7 cells and S3 tumorspheres. Since a possible involvement of $\mathrm{BC}$ stem cells has been postulated in anti-estrogen resistance 
A

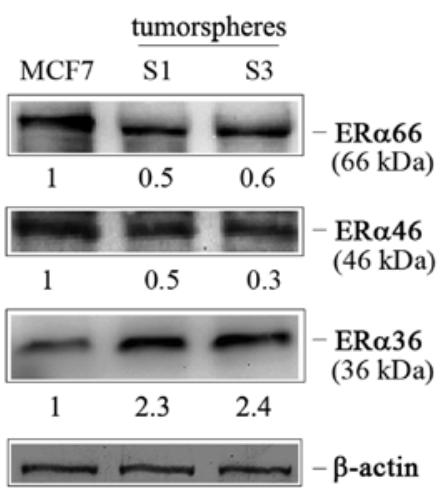

B

\begin{tabular}{|c|c|c|c|c|c|c|}
\hline \multicolumn{3}{|c|}{ MCF7 } & \multicolumn{3}{|c|}{ S3 tumorspheres } & \\
\hline- & + & - & - & + & - & $10 \mathrm{nM}$ E2 \\
\hline - & - & + & - & - & + & $25 \mu \mathrm{M}$ genistein \\
\hline$\longrightarrow$ & $=$ & $=$ & - & $\longrightarrow$ & $m$ & $-E R \alpha 66$ \\
\hline 1 & 1.5 & 1.6 & 0.5 & 0.4 & 0.3 & $\mathrm{kDa})$ \\
\hline- & $\longrightarrow$ & - & - & - & - & $-E R \alpha 36$ \\
\hline 1 & 0.9 & 0.8 & 1.6 & 2.1 & 1.9 & $(2,10$ \\
\hline 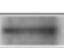 & 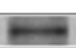 & $\Leftrightarrow$ & 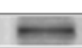 & 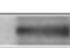 & $\longrightarrow$ & $-\beta$-actin \\
\hline
\end{tabular}

Figure 3. Comparative analysis of ER $\alpha$ isoforms in adherent MCF7 cells and tumorspheres. (A) Immunoblotting of ER $\alpha$ receptor isoforms. Cells and tumorspheres (S1 and S3) were lysed and protein extracts were submitted to western blot analysis as reported in Materials and methods. (B) Effect of estrogen treatment on ER $\alpha$ variants. MCF7 cells and tumorspheres were exposed to hormone treatment ( $10 \mathrm{nM}$ E2 or $25 \mu \mathrm{M}$ genistein) for $48 \mathrm{~h}$. Then cells were collected, lysed and subjected to western blot analysis. The correct amount of proteins onto the gel was checked by immunoblotting for $\beta$-actin The images are representative of three independent experiments with similar results.

of ER-positive BC (25), we aimed to clarify the estrogen responsiveness of $\mathrm{BC}$ tumorspheres. At first, we treated S3 tumorspheres with $17-\beta$ estradiol (E2) or genistein, a natural isoflavonoid phytoestrogen that possesses BC preventive properties (26). The doses (10 nM E2 or $25 \mu \mathrm{M}$ genistein) employed for these studies were chosen on the basis of preliminary experiments demonstrating that the incubation of tumorspheres or parental MCF7 cells with different concentrations (100 pM-100 $\mu \mathrm{M})$ of hormones for $48 \mathrm{~h}$ increased cell number at low doses while reduced cell viability at higher concentrations (50-100 $\mu \mathrm{M})$ (not shown). In light of these data we evaluated the effect of the hormones in S3 tumorspheres seeded in low attachment conditions as reported in Materials and methods. As shown in Fig. 2A and $\mathrm{B}$, the treatment increased $\mathrm{S} 3$ tumorsphere population by $45 \%$ with E2 and by $66 \%$ with genistein at $48 \mathrm{~h}$. Moreover, a remarkable increase in tumorsphere sizes (Fig. 2C) was also observed. Approximately $86 \%$ of E2-treated tumorspheres showed a diameter $>50 \mu \mathrm{m}$, most of which with a mean diameter of $100-150 \mu \mathrm{m}$. This percentage amounted to $98 \%$ in genistein-treated tumorspheres, showing an enrichment in the population with a mean diameter of 150-200 $\mu \mathrm{m}$.

The effect of estrogens on cell viability of S3 tumorspheres was also analyzed by MTT assay. As Fig. 2D reports, the incubation with low doses of hormones stimulated the growth of S3 tumorspheres by about 50\% with E2 and by $63 \%$ with genistein, values which were higher than those obtained in MCF7 cells.

In accordance with these results, western blot analyses provided evidence that, albeit S3 tumorspheres expressed a lower level of the proliferating marker PCNA in comparison to parental MCF7 cells, it significantly increased after E2 or genisten stimulation (Fig. 2E).

Intrigued by these results we focused on $\mathrm{ER} \alpha$ receptor isoforms which mediate estrogen effects (27). Considering the different sensitivity of MCF7 cells and S3 tumorspheres to both E2 and genistein, we wondered whether the observed effects were linked to a different expression of ER receptors. Thus, we evaluated the presence of the isoforms of ER $\alpha$ (ER $\alpha 66$, its splice variant ER $\alpha 46$, and the recently identified variant ERa36) in $S 1$ and $S 3$ tumorspheres in comparison to MCF7 cells. As Fig. 3A shows, tumorspheres expressed a very low amount of ER $\alpha 66$ and ER $\alpha 46$ isoforms respect to MCF7 cells, while a remarkable expression of ER $\alpha 36$ isoform was visible. Moreover, the exposure of S3 tumorspheres to estrogen treatment (E2 and genistein) further increased ER $\alpha 36$ content reducing that of ER $\alpha 66$. Such an effect was dissimilar to that observed in parental MCF7 cells (Fig. 3B), where hormone treatment increased the ERa66 level.

Breast cancer tumorspheres express high levels of serpin proteinase inhibitor 9. In light of the results obtained on $\mathrm{ER} \alpha$ receptor isoforms, our attention was next focused on PI-9 (proteinase inhibitor 9), a member of serpin family which is subjected to the hormonal induction mediated by ER $\alpha 66$-dependent transcriptional regulation (28). Since S3 tumorspheres showed a higher expression of ER $\alpha 36$ receptor which lacks transcriptional activity, we expected to find a lower level of PI-9 in tumorspheres than parental MCF7 cells. Surprisingly, western blot analysis revealed that the level of PI-9 protein was 1.6 and 3.5-fold higher in S1 and S3 tumorspheres, respectively (Fig. 4A). Based on these data, we wondered whether this upregulated level could be ascribed to an increase in mRNA level. To verify this hypothesis, we performed comparative analyses by real-time RT-PCR. Data reported in Fig. 4B revealed that S3 tumorspheres also expressed a pronounced level of PI-9 mRNA which was 110-fold higher than that found in adherent MCF7 cells, while a very modest increase of this factor was observed in S1 tumorspheres.

Interestingly, $17-\beta$ estradiol or genistein treatment further increased the level of PI-9, thus indicating a possible relationship between PI-9 expression and estrogen-mediated signaling (Fig. 4C).

S3 tumorspheres show an active CXCR4/phospho-p38 proliferative axis. Next, we explored possible molecular scenarios responsible for PI-9 upregulation in S3 tumorspheres. In this regard, we focused particular attention on CXCR4, the chemokine receptor specific for the stromal-derived-factor-1 (29). This receptor represents a prognostic factor in different types of cancer and plays a role in chemotaxis, stemness and drug resistance $(30,31)$. Moreover, recent data have also described this factor as a key regulator of breast cancer invasion, directing 
A
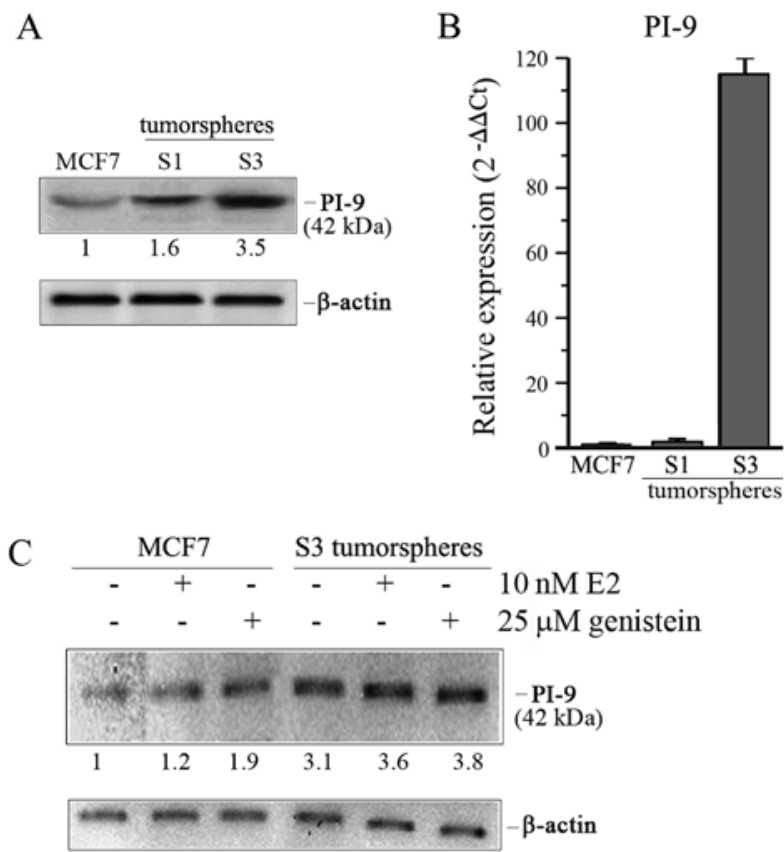

Figure 4. Analysis of proteinase inhibitor PI-9 in adherent MCF7 cells and tumorspheres. (A) Immunoblotting of PI-9 level. Cells and tumorspheres were lysed and protein extracts were submitted to western blot analysis as reported in Materials and methods. (B) Quantitative RT-PCR of PI-9 in adherent MCF7 and tumorspheres. mRNA from MCF7 cells and tumorspheres was reverse-transcribed as reported in Materials and methods and gene expression was analyzed by real-time PCR. (C) Effect of E2 or genistein treatment on PI-9 protein content. After exposure of MCF7 cells and tumorspheres to hormone treatment for $48 \mathrm{~h}$, cells were collected, lysed and subjected to western blot analysis. In (A) and (C) the correct amount of proteins onto the gel was checked by immunoblotting for $\beta$-actin. In (B) error bars depict standard deviations. All experiments were performed in triplicates.

A

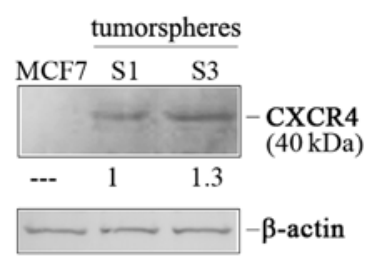

$\mathrm{C}$

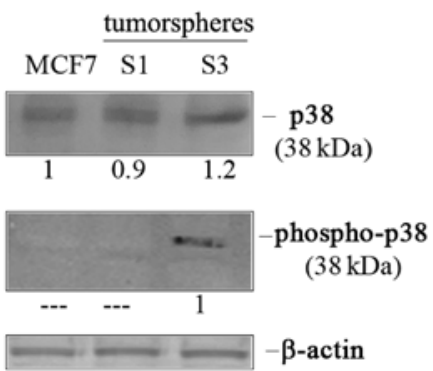

Figure 5. Analysis of CXCR4, p38 and phospho-p38 in MCF7 cells and BC tumorspheres. Western blot analysis (A) and real-time PCR (B) of CXCR4 expression in MCF7 cells and S1 and S3 tumorspheres. (C) Immunoblotting of p38 and phospho-p38 by western blotting as reported in Materials and methods. In (A) and (C) the correct amount of proteins onto the gel was checked by immunoblotting for $\beta$-actin. In (B) error bars depict standard deviations. All experiments were performed in triplicates.

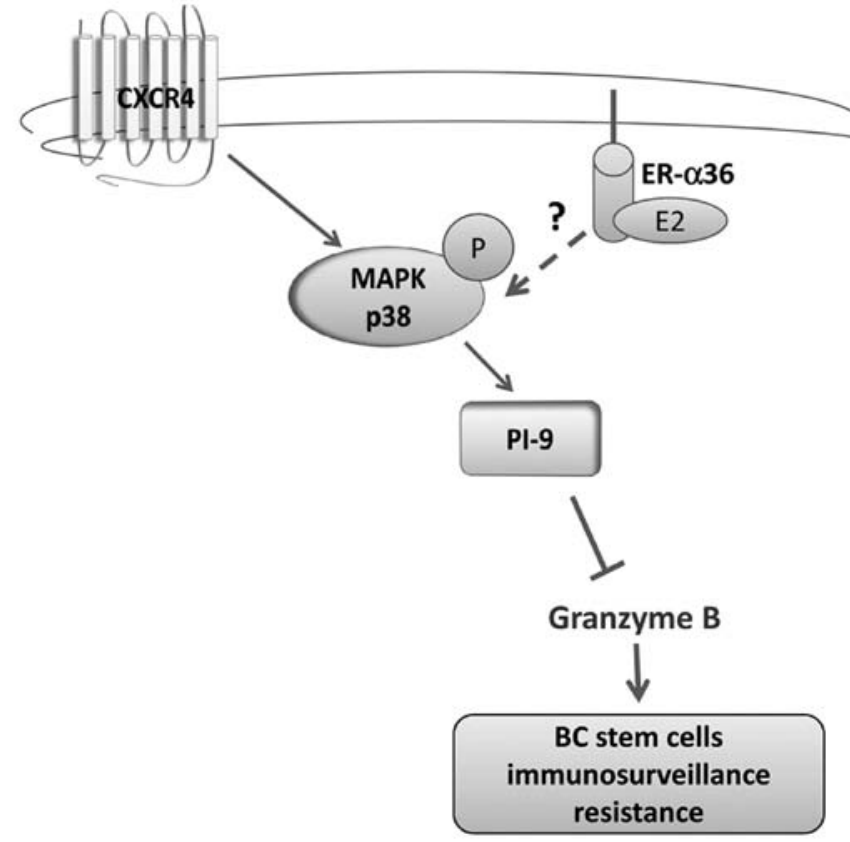

Figure 6. Schematic representation of the possible mechanism of action controlling the high PI-9 levels in BC tumorspheres.

homing of BC cells to particular sites of metastases (32). In our experiments we observed that CXCR4 was undetectable in parental MCF7 cells, while it appeared in tumorspheres increasing from $\mathrm{S} 1$ to $\mathrm{S} 3$ generation (Fig. 5A). Accordingly, mRNA analysis by real-time PCR showed a 77.7-fold higher content in S3 tumorspheres than MCF7 cells (Fig. 5B).

Since Bots et al (33) have shown that PI-9 is induced through p38 MAP kinase activation in dendritic cells and the activation of this kinase can be linked to CXCR4, we analyzed the level of p38 kinase in S3 tumorspheres. As shown in Fig. 5C, we demonstrated that $\mathrm{p} 38$ was present in both parental MCF7 cells and tumorspheres, but its content was only 1.2-fold higher in S3 tumorspheres than parental cells. Interestingly, the phosphorylated and active form of the protein, detected by using an antibody directed against the dual phosphorylated sites Thr180 and Tyr182, was found only in S3 tumorspheres (Fig. 5C), indicating the presence of an active p38 MAP kinase pathway in this stem-like population.

\section{Discussion}

Cytotoxic T lymphocytes (CTL) and natural killer (NK) cells can specifically identify and eliminate tumor cells in a process termed cancer immunosurveillance that exerts a crucial role in inhibiting carcinogenesis and maintaining cellular homeostasis (34). Unfortunately, tumor cells develop multiple strategies to evade host immune responses, including reduced expression of major histocompatibility complex molecules and loss of tumor antigens (35). Recently, it has been suggested that the inhibition of granzyme B activity in neoplastic cells may constitute one of the mechanisms that tumors use to escape immunosurveillance (36,37). Granzyme B belongs to a group of serine proteases commonly found in the cytotoxic granules of CTL and NK cells and it mediates apoptosis in target cells together with the pore forming protein perforin 
(38). The proteinase inhibitor 9 (PI-9), a member of family of serine proteinase inhibitors, is a direct inhibitor of granzyme B in human (39). Its overexpression has been observed in several types of cancer, such as breast (28), prostate (40) and lung cancer (41) and has been correlated to the protection of tumor cells from granzyme B-mediated cytotoxicity. However, no data concerning the status of PI-9 in cancer stem cells are available so far.

In this study we employed tumorspheres obtained by us from MCF7 cells serially passaged up to the third generation (S3 tumorspheres) and expressing high levels of stem cell markers. Interestingly, we show for the first time that $E R \alpha^{+}$breast cancer tumorspheres express higher levels of PI-9 protein than parental MCF7 cells and the content of this serpin proteinase inhibitor progressively increases with the passaging, resulting more pronounced in S3 tumorspheres. Real-time RT-PCR also provided evidence that the increased expression of PI-9 is related to gene upregulation.

It has been shown that estrogens increase PI-9 level through an ERa66-dependent transcriptional regulation in breast cancer cells (28). In particular, in PI-9 promoter it has been identified a sequence located $200 \mathrm{bp}$ downstream of the transcription start site and binding the estrogen/ER $\alpha$ complex (27). These observations prompted us to evaluate a possible involvement of estrogens in PI-9 induction in MCF7-derived tumorspheres. After proving that estrogens (17- $\beta$ estradiol and genistein) increase both the number and sizes of tertiary tumorspheres as well as the level of proliferation marker PCNA, we demonstrated that both the hormones markedly increase PI-9 level in tumorspheres, suggesting their involvement in PI-9 upregulation. Estrogens are known to regulate different cellular processes stimulating both genomic and non-genomic pathways (3-8). The classical mechanism of estrogen action involves the binding of the hormones to specific nuclear receptors (ER $\alpha 66$, its splice variant ER $\alpha 46$, and the closely related estrogen receptor ER $\beta)(7,8)$ which bind to estrogen responsive elements (EREs) located in the promoter of target genes (8). The experiments described here demonstrated that the levels of both ER $\alpha 66$ and ER $\alpha 46$ are lower in tertiary tumorspheres than in parental MCF7 cells. Moreover, both 17- $\beta$ estradiol and genistein further decreased the level of ER $\alpha 66$ isoform. The divergent trends between the increase in PI-9 and the decrease in ER $\alpha 66$ observed in tertiary tumorspheres seem to indicate that ER $\alpha 66$ isoform is not involved in PI-9 upregulation in $\mathrm{ER} \alpha$-positive $\mathrm{BC}$-derived spheres.

Estrogens were also reported to bind cell surface receptors to transduce membrane-initiated estrogen signaling (7). These effects seem to be related to the expression of ER $\alpha 36$, a variant of ER $\alpha 66$ (9) which lacks both the transcriptional activation domains (AF-1 and AF-2). Being mainly located near the plasma membrane and in the cytoplasm, it mediates the activation of MAPK/ERK and PI3K/AKT signaling pathways $(42,43)$. Our results showed that ER $\alpha 36$ is expressed at a higher level in tertiary tumorspheres than MCF7 cells and its content is further increased by $17-\beta$ estradiol or genistein treatment. These results are in accordance with the observation of Deng et al (44) showing that ER $\alpha 36$ expression is markedly increased in stem cells derived from ER-positive breast cancer cells where it plays an important role in expanding breast cancer stem-like cell population. Thus, we postulated that the activation of non-genomic signaling involving estrogen/ ER $\alpha 36$ could be responsible for PI-9 increased expression in tertiary tumorspheres. This hypothesis is also corroborated by the observation that ER $\alpha 36$ stimulation is involved in the activation of MAPK/ERK signaling pathway (45) and that the expression of PI-9 is regulated via the p38/MAPK pathway in human dendritic cells (33). Interestingly, we demonstrate that both parental MCF7 cells and tertiary tumorspheres express $\mathrm{p} 38$, while the phosphorylated and active form of this protein only occurs in tumorspheres of third generation, thus suggesting that this event could be related to the increase in PI-9 content found in tertiary tumorspheres.

Recently, several lines of evidence suggested that the biological effects of CXCR4, the chemokine receptor specific for the stromal-derived-factor-1 (SDF-1), are mediated by the activation of MAPK family members (46). The SDF-1-CXCR4 signaling axis is a known mediator of metastasis in breast cancer (32) as demonstrated by CXCR4 expression observed in human breast cancer specimens (47). A recent study has also shown that CXCR4 maintains a cancer progenitor population in tamoxifen-resistant breast cancer cells (48). Data reported herein showing that tertiary tumorspheres express high levels of both CXCR4 mRNA and protein suggest that the activation of this receptor could be also involved in p38 MAPK activation.

Taken together, our results demonstrate for the first time that $\mathrm{ER} \alpha^{+}$breast cancer stem-like cells express a high content of granzyme B inhibitor PI-9 and that this event could be ascribed to the activation of a CXCR4-p38 axis. Considering that $\mathrm{ER} \alpha 36$ has been reported to play a role in MAPK signaling activation (43), it is plausible to hypothesize an involvement of ER $\alpha 36$ isoform which is overexpressed in tertiary tumorspheres in this signaling circuit. A possible molecular mechanism controlling this pathway in $\mathrm{BC}$ tumorspheres is illustrated in Fig. 6.

In conclusion, we consider that PI-9 overexpression found in mammary tumorspheres could cause a short-circuit in immunosurveillance's signaling preserving these cells from cytotoxic T lymphocytes and NK cell-mediated apoptosis. Therefore, this experimental system might represent a suitable in vitro model to study breast cancer-initiating cells and to explore the response of these tumorspheres to granzyme B and agents molecularly interfering with immunosurveillance system.

\section{Acknowledgements}

This study was supported by a grant from: European Regional Development Fund, European Territorial Cooperation 2007-2013, CCI 2007 CB 163 PO 037, OP Italia-Malta 2007-2013; Dr D. Carlisi was supported by a grant by 'Italian Ministry of Education, University and Research (MIUR)'. Dr C. Cernigliaro was financially supported by a research contract of the OP Italia-Malta 2007-2013 grant.

\section{References}

1. Forouzanfar MH, Foreman KJ, Delossantos AM, Lozano R, Lopez AD, Murray CJ and Naghavi M: Breast and cervical cancer in 187 countries between 1980 and 2010: A systematic analysis. Lancet 378: 1461-1484, 2011. 
2. Key T, Appleby P, Barnes I, Reeves G; Endogenous Hormones and Breast Cancer Collaborative Group: Endogenous sex hormones and breast cancer in postmenopausal women: reanalysis of nine prospective studies. J Nat Cancer Inst 94: 606e616, 2002.

3. Welboren WJ, Sweep FC, Span PN and Stunnenberg HG: Genomic actions of estrogen receptor alpha: What are the targets and how are they regulated? Endocr Relat Cancer 16: 1073-1089, 2009.

4. Robertson JF: Oestrogen receptor: A stable phenotype in breast cancer. Br J Cancer 73: 5-12, 1996.

5. Nilsson S, Mäkelä S, Treuter E, Tujague M, Thomsen J, Andersson G, Enmark E, Pettersson K, Warner M and Gustafsson JA: Mechanisms of estrogen action. Physiol Rev 81: $1535-1565,2001$.

6. Katzenellenbogen BS and Katzenellenbogen JA: Estrogen receptor transcription and transactivation: Estrogen receptor alpha and estrogen receptor beta: regulation by selective estrogen receptor modulators and importance in breast cancer. Breast Cancer Res 2: 335-344, 2000.

7. Björnström L and Sjöberg M: Mechanisms of estrogen receptor signaling: Convergence of genomic and nongenomic actions on target genes. Mol Endocrinol 19: 833-842, 2005.

8. Deroo BJ and Buensuceso AV: Minireview: Estrogen receptorbeta: mechanistic insights from recent studies. Mol Endocrino 24: 1703-1714, 2010.

9. Wang Z, Zhang X, Shen P, Loggie BW, Chang Y and Deuel TF: Identification, cloning, and expression of human estrogen receptor-alpha36, a novel variant of human estrogen receptoralpha66. Biochem Biophys Res Commun 336: 1023-1027, 2005.

10. Chaudhri RA, Schwartz N, Elbaradie K, Schwartz Z and Boyan BD: Role of ER $\alpha 36$ in membrane-associated signaling by estrogen. Steroids 81: 74-80, 2014.

11. Zou Y, Ding L, Coleman M and Wang Z: Estrogen receptor-alpha (ER-alpha) suppresses expression of its variant ER-alpha 36 FEBS Lett 583: 1368-1374, 2009.

12. Osborne CK: Tamoxifen in the treatment of breast cancer. N Eng J Med 339: 1609-1618, 1998.

13. Clarke R, Leonessa F, Welch JN and Skaar TC: Cellular and molecular pharmacology of antiestrogen action and resistance. Pharmacol Rev 53: 25-71, 2001.

14. Clarke R, Liu MC, Bouker KB, Gu Z, Lee RY, Zhu Y, Skaar TC, Gomez B, O'Brien K, Wang Y, et al: Antiestrogen resistance in breast cancer and the role of estrogen receptor signaling. Oncogene 22: 7316-7339, 2003

15. Charafe-Jauffret E, Ginestier C, Iovino F, Wicinski J, Cervera N, Finetti P, Hur MH, Diebel ME, Monville F, Dutcher J, et al: Breast cancer cell lines contain functional cancer stem cells with metastatic capacity and a distinct molecular signature. Cancer Res 69: 1302-1313, 2009.

16. Deng H, Yin L, Zhang XT, Liu LJ, Wang ML and Wang ZY: ER-o variant ER- $\alpha 36$ mediates antiestrogen resistance in ER-positive breast cancer stem/progenitor cells. J Steroid Biochem Mol Biol 144B: 417-426, 2014.

17. Massari F, Santoni M, Ciccarese C and Santini D: The immunocheckpoints in modern oncology: The next 15 years. Expert Opin Biol Ther 15: 917-921, 2015.

18. Mori S, Jewett A, Murakami-Mori K, Cavalcanti M and Bonavida B: The participation of the Fas-mediated cytotoxic pathway by natural killer cells is tumor-cell-dependent. Cancer Immunol Immunother 44: 282-290, 1997.

19. Goping IS, Barry M, Liston P, Sawchuk T, Constantinescu G, Michalak KM, Shostak I, Roberts DL, Hunter AM, Korneluk R, et al: Granzyme B-induced apoptosis requires both direct caspase activation and relief of caspase inhibition. Immunity 18 : 355-365, 2003

20. Fritsch K, Finke J and Grüllich C: Suppression of granzyme B activity and caspase-3 activation in leukaemia cells constitutively expressing the protease inhibitor 9. Ann Hematol 92: 1603-1609, 2013.

21. Dontu G, Abdallah WM, Foley JM, Jackson KW, Clarke MF, Kawamura MJ and Wicha MS: In vitro propagation and transcriptional profiling of human mammary stem/progenitor cells. Genes Dev 17: 1253-1270, 2003.

22. D'Anneo A, Carlisi D, Lauricella M, Puleio R, Martinez R, Di Bella S, Di Marco P, Emanuele S, Di Fiore R, Guercio A, et al: Parthenolide generates reactive oxygen species and autophagy in MDA-MB231 cells. A soluble parthenolide analogue inhibits tumour growth and metastasis in a xenograft model of breast cancer. Cell Death Dis 4: e891, 2013.
23. Di Fiore R, Drago-Ferrante R, Pentimalli F, Di Marzo D, Forte IM, D'Anneo A, Carlisi D, De Blasio A, Giuliano M, Tesoriere G, et al: MicroRNA-29b-1 impairs in vitro cell proliferation, self-renewal and chemoresistance of human osteosarcoma 3AB-OS cancer stem cells. Int J Oncol 45: 2013-2023, 2014.

24. Carlisi D, D'Anneo A, Martinez R, Emanuele S, Buttitta G, Di Fiore R, Vento R, Tesoriere G and Lauricella M: The oxygen radicals involved in the toxicity induced by parthenolide in MDA-MB-231 cells. Oncol Rep 32: 167-172, 2014.

25. Arif K, Hussain I, Rea C and El-Sheemy M: The role of Nanog expression in tamoxifen-resistant breast cancer cells. Onco Targets Ther 8: 1327-1334, 2015.

26. Bilal I, Chowdhury A, Davidson J and Whitehead S: Phytoestrogens and prevention of breast cancer: The contentious debate. World J Clin Oncol 5: 705-712, 2014.

27. Krieg AJ, Krieg SA, Ahn BS and Shapiro DJ: Interplay between estrogen response element sequence and ligands controls in vivo binding of estrogen receptor to regulated genes. J Biol Chem 279: 5025-5034, 2004

28. Jiang X, Ellison SJ, Alarid ET and Shapiro DJ: Interplay between the levels of estrogen and estrogen receptor controls the level of the granzyme inhibitor, proteinase inhibitor 9 and susceptibility to immune surveillance by natural killer cells. Oncogene 26: 4106-4114, 2007.

29. Furusato B, Mohamed A, Uhlén M and Rhim JS: CXCR4 and cancer. Pathol Int 60: 497-505, 2010.

30. Trautmann F, Cojoc M, Kurth I, Melin N, Bouchez LC, Dubrovska A and Peitzsch C: CXCR4 as biomarker for radioresistant cancer stem cells. Int J Radiat Biol 90: 687-699, 2014.

31. Lourenco S, Teixeira VH, Kalber T, Jose RJ, Floto RA and Janes SM: Macrophage migration inhibitory factor-CXCR4 is the dominant chemotactic axis in human mesenchymal stem cell recruitment to tumors. J Immunol 194: 3463-3474, 2015.

32. Rhodes LV, Short SP, Neel NF, Salvo VA, Zhu Y, Elliott S, Wei Y, Yu D, Sun M, Muir SE, et al: Cytokine receptor CXCR4 mediates estrogen-independent tumorigenesis, metastasis, and resistance to endocrine therapy in human breast cancer. Cancer Res 71: 603-613, 2011

33. Bots M, de Bruin E, Rademaker-Koot MT and Medema JP: Proteinase inhibitor-9 expression is induced by maturation in dendritic cells via p38 MAP kinase. Hum Immunol 68: 959-964, 2007.

34. Lakshmi Narendra B, Eshvendar Reddy K, Shantikumar S and Ramakrishna S: Immune system: A double-edged sword in cancer. Inflamm Res 62: 823-834, 2013.

35. Krasnova Y, Putz EM, Smyth MJ and Souza-FonsecaGuimaraes F: Bench to bedside: NK cells and control of metastasis. Clin Immunol 15: 30050-30054, 2015.

36. Medema JP, de Jong J, Peltenburg LT, Verdegaal EM, Gorter A, Bres SA, Franken KL, Hahne M, Albar JP, Melief CJ, et al: Blockade of the granzyme B/perforin pathway through overexpression of the serine protease inhibitor PI-9/SPI-6 constitutes a mechanism for immune escape by tumors. Proc Natl Acad Sci USA 98: 11515-11520, 2001.

37. Igney $\mathrm{FH}$ and Krammer PH: Immune escape of tumors: Apoptosis resistance and tumor counterattack. J Leukoc Biol 71: 907-920, 2002

38. Martínez-Lostao L, Anel A and Pardo J: How do cytotoxic lymphocytes kill cancer cells? Clin Cancer Res 21: 5047-5056, 2015.

39. Cunningham TD, Jiang $X$ and Shapiro DJ: Expression of high levels of human proteinase inhibitor 9 blocks both perforin/ granzyme and Fas/Fas ligand-mediated cytotoxicity. Cell Immunol 245: 32-41, 2007.

40. Ray M, Hostetter DR, Loeb CR, Simko J and Craik CS: Inhibition of Granzyme B by PI-9 protects prostate cancer cells from apoptosis. Prostate 72: 846-855, 2012.

41. Soriano C, Mukaro V, Hodge G, Ahern J, Holmes M, Jersmann H, Moffat D, Meredith D, Jurisevic C, Reynolds PN, et al: Increased proteinase inhibitor-9 (PI-9) and reduced granzyme B in lung cancer: Mechanism for immune evasion? Lung Cancer 77: 38-45, 2012.

42. Levin ER: Cellular functions of plasma membrane estrogen receptors. Steroids 67: 471-475, 2002.

43. Lee LM-J, Cao J, Deng H, Chen P, Gatalica Z and Wang ZY: ER-alpha36, a novel variant of ER-alpha, is expressed in ER-positive and -negative human breast carcinomas. Anticancer Res 28B: 479-483, 2008. 
44. Deng H, Zhang XT, Wang ML, Zheng HY, Liu LJ and Wang ZY: ER-a36-mediated rapid estrogen signaling positively regulates ER-positive breast cancer stem/progenitor cells. PLoS One 9: e88034, 2014.

45. Zhang X, Deng H and Wang ZY: Estrogen activation of the mitogen-activated protein kinase is mediated by ER- $\alpha 36$ in ER-positive breast cancer cells. J Steroid Biochem Mol Biol 143: 434-443, 2014

46. Mao L, Yuan L, Slakey LM, Jones FE, Burow ME and Hill SM: Inhibition of breast cancer cell invasion by melatonin is mediated through regulation of the p38 mitogen-activated protein kinase signaling pathway. Breast Cancer Res 12: R107, 2010.
47. Zhang M, Liu HX, Teng XD, Wang HB, Cui J, Jia SS, Gu XY and Li ZG: The differences in CXCR4 protein expression are significant for the five molecular subtypes of breast cancer. Ultrastruct Pathol 36: 381-386, 2012.

48. Dubrovska A, Hartung A, Bouchez LC, Walker JR, Reddy VA, Cho CY and Schultz PG: CXCR4 activation maintains a stem cell population in tamoxifen-resistant breast cancer cells through AhR signalling. Br J Cancer 107: 43-52, 2012. 